\title{
PERSPECTIVES D'UTILISATION DE ECHINOSTOMA TOGOENSIS JOURDANE ET KULO, 1981 DANS LE CONTROLE BIOLOGIQUE DE LA BILHARZIOSE INTESTINALE EN AFRIQUE
}

\author{
J. JOURDANE* et S. D. KULO**
}

RÉSUMÉ. L'étude du développement expérimental de Echinostoma togoensis Jourdane et Kulo, 1981 chez ses hôtes successifs a permis de montrer que cette espèce associe un certain nombre de caractères susceptibles d'être utilisés en vue d'un contrôle biologique de la bilharziose intestinale :

— effet stérilisant total et durable à l'égard de Biomphalaria pfeifferi;

— pathogénicité vis-à-vis du vecteur lors des infestations avec plus de 5 miracidiums ;

- dominance très marquée sur les stades intramolluscaux de Schistosoma mansoni;

— facilité et faible coût d'entretien du cycle (le parasite évolue chez deux hôtes communs : le Planorbe et la Souris) ;

— forte productivité en œufs chez la Souris (10 Souris infestées avec 75 métacercaires assurent une production journalière moyenne de 620000 œufs.)

Perspectives of the use of Echinostoma togoensis Jourdane et Kulo, 1981 for biological control of intestinal schistosomiasis in Africa.

SUMMARY. A study of the experimental development of E. togoensis Jourdane et Kulo, 1981 in its successive hosts has allowed us to show that this species combines several characteristics considered to be essential with a view to biological control of intestinal schistosomiasis:

- a complete and permanent sterilizing effect of Biomphalaria pfeifferi;

- a pathogenicity with respect to the molluse in infestations with more than 5 miracidia ;

- a very clear dominance of intramolluscal stages of Schistosoma mansoni;

- ease and low cost of cycle maintenance (the parasite develops in two common hosts: the planorb and the mouse;

- a high egg productivity in the mouse $(10$ mice infected with 75 metacercariea produce on average 620.000 eggs per day.

L'idée d'utiliser les Trématodes dans le contrôle biologique des bilharzioses humaines est ancienne. Bayer, dès 1954, avait envisagé un tel mode de lutte pour divers vecteurs de bilharzioses. Ce n'est cependant qu'au cours de la dernière décennie que des recherches expérimentales précises et surtout des essais sur le terrain ont été réalisés. Dans une récente revue sur les travaux entrepris jusqu'ici dans le domaine

* Département de Biologie animale (Directeur: Pr. C. Combes), Université, F 66025 Perpignan Cedex.

** Laboratoire de Zoologie, École des Sciences, BP 1515 Lomé (Togo).

Accepté le 22 mars 1982. 
de la lutte biologique par Trématodes, Combes (1981) analyse avec précision l'ensemble des problèmes posés par cette voie de contrôle et met l'accent sur les recherches à poursuivre.

Nous avons eu l'occasion d'isoler récemment au Togo un Échinostome nouveau, Echisnostoma togoensis Jourdane et Kulo, 1981, dont les particularités du développement chez le Mollusque premier hôte et chez le Vertébré ouvrent des perspectives prometteuses d'utilisation de ce parasite en lutte biologique. Nous présentons ici le résultat des recherches sur le développement de cette espèce.

\section{Matériel et méthodes}

Le cycle de $E$. togoensis ne pose aucun problème de maintenance au laboratoire. Le parasite évolue au stade rédie et au stade métacercaire chez les Mollusques Pulmonés Biomphalaria glabrata et Biomphalaria pfeifferi et au stade adulte chez la Souris blanche. Les études sur l'effet stérilisant ont été faites sur des B. pfeifferi d'origine togolaise.

Les techniques d'infestation des Mollusques ( $1^{\mathrm{er}}$ ou $2^{\mathrm{e}}$ hôte intermédiaire) sont classiques : les Mollusques sont exposés individuellement dans des piluliers contenant 5 cc d'eau de source à des miracidiums ou des cercaires fraîchement émis ; le nombre de larves infestantes est variable en fonction du protocole expérimental.

Les Souris sont infestées par tubage gastrique sans anesthésie.

Les œufs produits par les adultes chez la Souris ont été récoltés selon la technique suivante : les Souris parasitées sont placées soit individuellement, soit par groupes, dans des cages à métabolisme (type IFFA CREDO) assurant la séparation des fèces et des urines. L'alimentation des animaux est donnée ad libitum. Les fèces sont recueillies le matin toutes les 24 heures. Dans un premier temps, les excréments sont dissociés par agitation pendant 15 minutes dans $250 \mathrm{cc}$ d'eau. Les œufs ainsi mis en suspension vont être ensuite séparés des résidus fécaux par tamisage sur une série de tamis à vide de maille décroissant $(500,250$ et $80 \mu \mathrm{m})$. Le filtrat enrichi en œufs est décanté plusieurs fois afin d'éliminer les suspensions colloïdales non retenues par les tamis. Les œufs sont finalement concentrés dans un volume d'eau égal à un litre et incubés à l'obscurité, à $26^{\circ}$, en enceinte climatique. L'éclosion des œufs est obtenue à partir du $13 \mathrm{e}$ jour. Le comptage des œufs a été fait à partir de 10 fractions aliquotes de $1 \mathrm{ml}$ de la dilution finale, chaque prélèvement étant effectué avec une pipette automatique. Les œufs sont comptés à la loupe binoculaire sur une cellule à numération globulaire.

\section{Pouvoir pathogène de E. togoensis sur B. pfeifferi}

\section{EfFet STÉRILISANT}

Cette étude a été entreprise d'une part chez des Mollusques n'ayant pas atteint la maturité sexuelle, d'autre part chez des Mollusques ayant commencé à pondre une semaine environ avant le jour de l'infestation. 
Dans chaque groupe, les expériences ont été réalisées sur plusieurs lots de Mollusques infestés par 5 miracidiums. Chaque lot de Mollusques a vu sa fécondité comparée à celle de lots témoins sains, composés d'un nombre identique de Mollusques de même taille.

Dans le premier groupe, la stérilisation du Mollusque est totale et définitive ; nous n'avons jamais observé de dépôt de pontes. Chez des lots de Mollusques gardés pendant plus de 6 mois, il n'y a pas eu de reprise de la fécondité.

Dans le deuxième groupe, comprenant des Planorbes ayant commencé à pondre au moment de l'infestation, la fécondité ne se maintient environ que jusqu'au $20^{\circ}$ jour. Ce délai correspond en gros à la durée de la période prépatente. Au-delà de cette période, la fécondité est supprimée de façon définitive.

Il apparaît donc que l'efficacité de la castration est totale dans les conditions expérimentales, quel que soit le stade physiologique du Mollusque au moment de l'infestation.

\section{RECHERCHES HISTOLOGIQUES SUR LES MÉCANISMES DE LA STÉRILISATION}

Les recherches ont été entreprises sur un lot de Mollusques mesurant entre 6 et $7 \mathrm{~mm}$ de diamètre au moment de l'infestation, donc ayant atteint la maturité sexuelle et sur un lot de Mollusques mesurant entre 2 et $3 \mathrm{~mm}$ de diamètre à l'infestation (donc au stade juvénile).

Les Mollusques des deux lots ont été infestés individuellement par 5 miracidiums. Deux Mollusques de chaque lot ont été fixés chaque semaine pendant 2 mois.

Les images histologiques observées ont été comparées à celles de lots témoins de Mollusques sains, de même taille et gardés dans les mêmes conditions de laboratoire.

Nous considérons les effets du parasite successivement dans les deux lots.

a - Action du parasite sur la gonade des Mollusques immatures.

Cette étude réalisée sur de très jeunes $B$. pfeifferi met en évidence un blocage absolu de l'activité gamétogénétique.

Dans les quelques rares îlots glandulaires non encore totalement détruits chez les Mollusques infestés depuis 20 jours, on peut vérifier que la gametogénèse ne se poursuit pas au-delà du stade spermatogonial. L'ovogenèse ne montre aucune image de croissance ovocytaire.

En ce qui concerne les organes sexuels secondaires (voies génitales et glandes annexes), ceux-ci apparaissent sous forme d'éléments vestigiaux quel que soit le stade de la parasitose.

\section{$\mathrm{b}$ - Action du parasite sur la gonade des Mollusques à maturité sexuelle:}

Dans un souci de clarté pour la compréhension des résultats, nous résumons les aspects pathogènes à partir d'observations faites au $19^{\mathrm{e}}$ jour et au $47^{\mathrm{e}}$ jour de la parasitose. 
Stade $19 \mathrm{e}$ jour :

A ce stade, la glande génitale du Mollusque est entièrement occupée par de nombreuses jeunes rédies-mères. La parasitose intéresse à un degré moindre la partie de la glande digestive postérieure à l'anse distale du tube digestif.

Les 2/3 postérieurs de la zone génitale sont vides de tout acinus sexuel. Les espaces primitivement occupés par l'ovotestis sont partiellement remplis de tissu connectif lâche montrant par places des accumulations de fibroblastes. Cette zone est le siège d'une castration totale du Mollusque par le Digène.

Seuls quelques rares îlots sexuels en voie de dégénérescence peuvent être dénombrés parmi de nombreuses rédies-filles dans le tiers proximal de la zone distale. A ce niveau néanmoins, l'architecture de la glande est profondément modifiée du fait de la compression mécanique exercée par l'intense mobilité des nombreuses rédies-filles. Les acini sexuels ont perdu leur aspect tubulaire et ne sont plus représentés que par de fins cordons à lumière souvent inexistante. En ce qui concerne la gamétogenèse, les observations suivantes peuvent être faites par comparaison avec les images histologiques fournies par les Mollusques témoins :

- l'épithélium germinatif ne montre pas de poussée gamétogénétique avec mise en place de nouvelles spermatogonies et ovogonies. Les images de mitoses y font totalement défaut. L'épithélium offre par contre tous les caractères d'un tissu dégénératif avec perte des contours cellulaires et pycnose accusée des noyaux ;

- tous les stades de la lignée mâle (spermatocyte, spermatide et spermatozoïde) ont disparu dans la lumière des acini ;

- quelques rares ovocytes sont reconnaissables dans certains îlots. Ceux-ci apparaissent profondément dégénérés avec une vacuolisation importante du cytoplasme.

Concernant les effets du parasitisme sur le tractus génital, ceux-ci n'apparaissent pas encore à l'évidence à ce stade. On note une réduction appréciable des digitations de la vésicule séminale où sont cependant visibles des spermatozoïdes non dégénérés.

Stade $47 \mathrm{e}$ jour :

La castration du Mollusque est ici totale. Aucun îlot germinal n'est identifiable dans les tissus distaux du Mollusque qui sont entièrement colonisés par les rédiesfilles du parasite. Les effets pathogènes du parasite gagnent aussi la zone distale de la glande digestive dont beaucoup de lobules glandulaires dégénèrent. Le tissu connectif développé par l'hôte au cours de la phase d'invasion parasitaire a également disparu dans toute la partie viscérale distale. Cette dernière apparaît sous| la forme d'une poche dans laquelle évolue une population très dense de rédies qui s'est totalement substituée aux viscères du Mollusque.

Les voies génitales sont le siège de modifications structurales et fonctionnelles très sensibles. Des réductions de taille intéressent pratiquement tous les organes sexuels accessoires : la glande mucipare, l'oothèque, la prostate, le préputium. Le réceptacle séminal et la vésicule séminale sont vides de spermatozoïdes.

L'activité secrétoire des glandes annexes (glande mucipare et prostate), que la coloration à l'Azan met particulièrement bien en évidence sur les lots témoins, a profondément régressé chez les Planorbes parasités. 


\section{c - Modalités d'action du parasite sur les organes sexuels du Mollusque:}

L'interprétation des images histologiques de la castration permet de conclure à un double mode d'action du parasite sur la glande sexuelle : une action indirecte et une action directe dont nous résumons les modalités :

- action indirecte :

Cet effet du parasite sur la glande sexuelle est évident car la phase initiale de la dégénérescence des éléments germinaux s'observe alors que l'intégrité de l'épithélium germinatif est encore entière. Cette action pourrait s'exercer grâce à des enzymes cytolytiques secrétées par les rédies et agissant spécifiquement sur les éléments germinaux du Mollusque, comme cela a été démontré pour d'autres Digènes (Cheng et Lee, 1968). Sluiters (1981) suggère l'existence d'une action indirecte du parasite sur les régulations endocrines ou neuro-endocrines du Mollusque responsables de la physiologie sexuelle. D'après nos observations, il apparaîtrait que les éléments germinaux de la lignée mâle sont plus sensibles à cette action indirecte puisque ceux-ci sont toujours détruits en premier.

Concernant les effets de la parasitose sur les organes sexuels secondaires, ceux-ci relèvent également d'un mécanisme indirect. Ils sont en effet la conséquence de la dégénérescence de l'ovotestis qui, dans les conditions normales, contrôle par voie hormonale leur différenciation.

- action directe :

Cette action ne peut être mise en évidence qu'à un stade déjà avancé de la dégénérescence de la glande sexuelle. Elle consiste en une atteinte directe de l'ovotestis par les rédies qui en dévorent les cellules en voie de lyse.

Si la présence d'éléments germinaux entiers est en effet exceptionnelle dans le tube digestif des rédies, des éléments cellulaires dissociés (noyaux, nucléoles, composants cytoplasmiques divers, pigments) y sont par contre systématiquement observés.

Les rédies ne sembleraient donc capables d'ingérer que des structures cellulaires très dégradées.

\section{EFFET LÉthaL}

A cette action stérilisante du Digène s'ajoute une action pathogène sur $B$. pfeifferi suggérée par nos expériences de stérilisation avec cinq miracidiums. Nous avons, en effet, été surpris de constater que l'évolution du parasite chez B. pfeifferi s'accompagne toujours d'une mortalité spectaculaire de l'hôte après la période prépatente. Il ne subsiste en moyenne que $50 \%$ des Mollusques après la $6^{\mathrm{e}}$ semaine, le reste de la population étant totalement décimé entre la $6^{\mathrm{e}}$ et la $8^{\mathrm{e}}$ semaine.

Dans le but de conforter cette observation, nous avons infesté plusieurs lots de $B$. pfeifferi avec un nombre plus élevé de miracidiums (10 et 20 miracidiums). Les courbes obtenues (fig. 1) montrent que des infestations plus fortes modifient de façon corrélative la courbe de survie des Mollusques. Avec 10 et 20 miracidiums, la demi-vie n'est que de 2 à 4 semaines en moyenne. A 6 semaines, plus aucun Mollusque vivant n'est dénombré dans les aquariums d'expérience. 


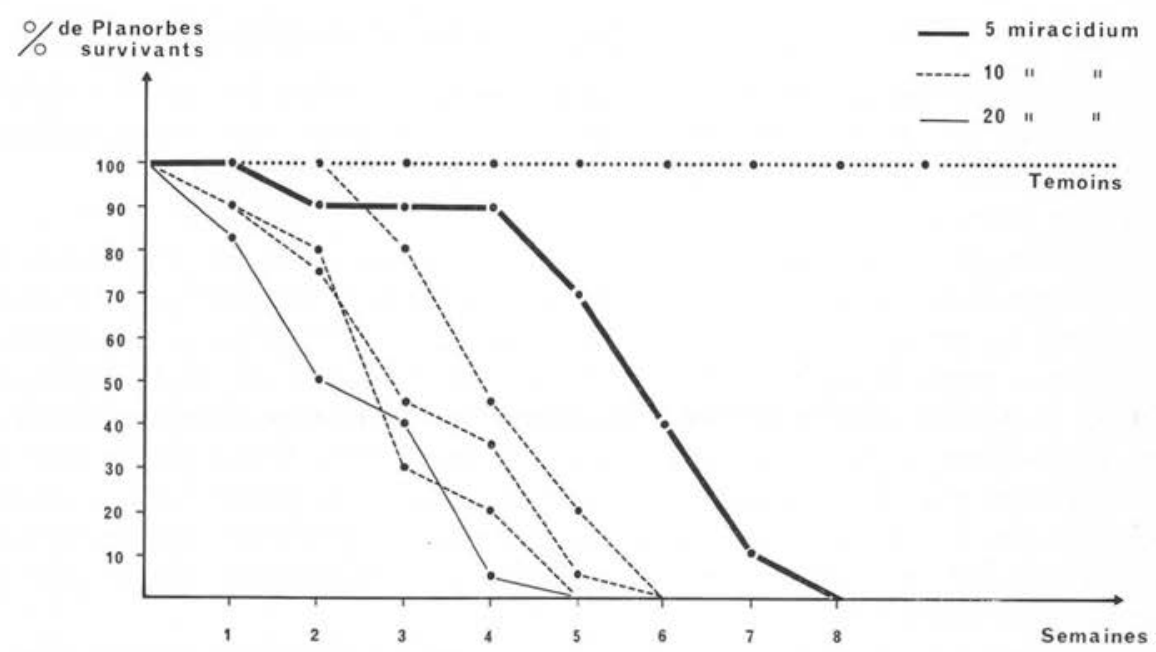

Fig. I. - E. togoensis. Courbes de survies comparées de B. pfeifferi infestés avec 5, ro ou 20 miracidiums de E. togoensis.

\section{Productivité d'E. togoensis chez la Souris}

La productivité a été testée à partir d'une série d'infestations réalisées avec des doses différentes de larves. Chaque expérience porte sur un lot de 4 Souris dont les fèces sont récoltées toutes les 24 heures à partir du $7 \mathrm{e}$ jour d'infestation, dans le but de dénombrer la totalité des œufs produits. La productivité a été ramenée pour chaque expérience au nombre d'œufs produits par parasite et par jour (o.p.j.), calculé à partir de la production totale de l'ensemble de la population parasitaire des 4 Souris.

Une première expérience réalisée sur des Souris hébergeant une population modérée de parasites (40 individus) nous a permis d'estimer les caractères à la fois qualitatifs et quantitatifs de la courbe de productivité.

Concernant les aspects qualitatifs, le graphique de la figure 2 montre que les premiers œufs apparaissent dans les selles dès le $9^{\mathrm{e}}$ jour d'infestation, et y sont dénombrés sans discontinuité jusqu'au 136e jour, date à laquelle nous avons disséqué les Souris afin de déterminer la population réelle de parasites. Nous avons pu vérifier, dans une autre expérience, que la fécondité du parasite se maintient pendant toute la durée de sa vie qui est de l'ordre de 6 mois.

Concernant les aspects quantitatifs, trois observations principales peuvent être faites :

- la productivité maximale journalière du parasite est très rapidement atteinte, dès le $19^{\mathrm{e}}$ jour ;

- le niveau moyen de production d'œufs par parasite et par jour pour toute la période de production est très élevée (840 o.p.j. dans notre expérience) ;

- la courbe de productivité ne correspond pas à un plateau mais témoigne à la fois 


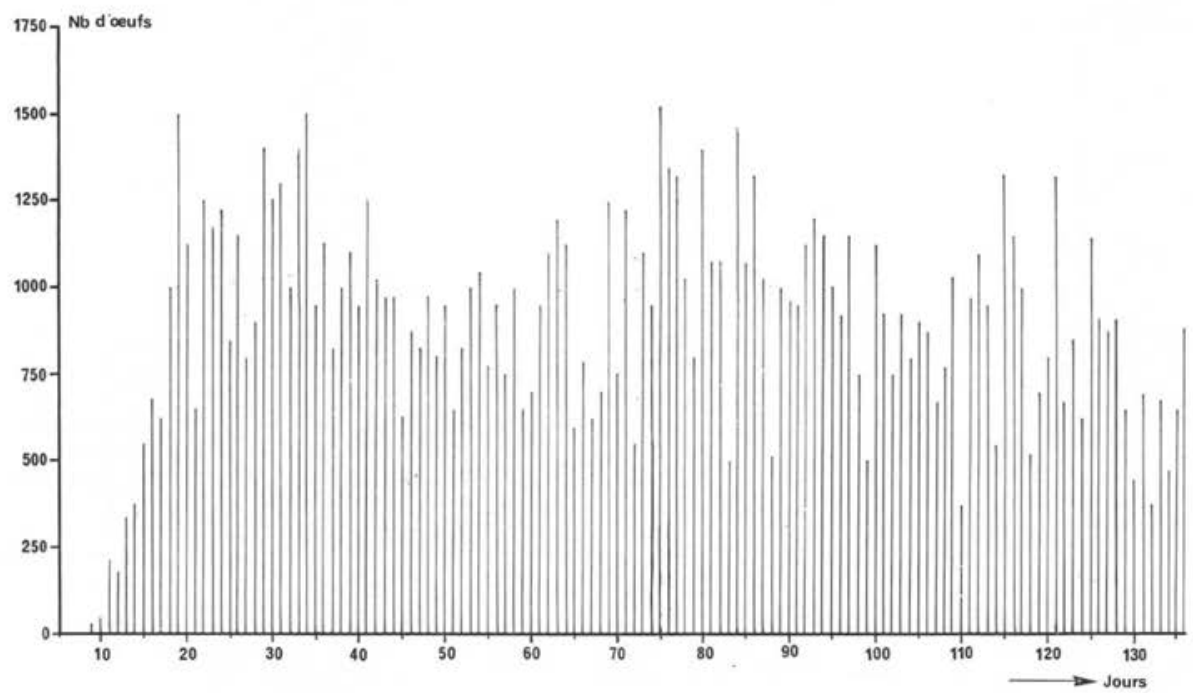

FIG. 2. - E. togoensis. Productivité journalière moyenne par parasite (expérience portant sur 4 Souris parasitées individuellement par 40 parasites).

de variations parfois très importantes d'un jour à l'autre et d'une alternance de périodes de forte et de faible productivité ; pour ce dernier caractère, une étude d'autocorrélation serait nécessaire pour déterminer l'échelle temporelle du rythme.

Sur la base de ces résultats expérimentaux, nous avons envisagé d'étudier l'influence de la densité de la population parasitaire sur la productivité individuelle : la productivité unitaire a été déterminée pour 1, 5, 75 et 150 parasites. Celle-ci a été appréciée dans le cadre de chaque expérience pendant 10 jours au moins.

Les résultats obtenus mettent en évidence une relation inversement proportionnelle entre la densité de la population et la productivité individuelle journalière. Celle-ci est minimum pour 150 parasites (360 o.p.j.) et maximum pour un parasite (1870 o.p.j.) (fig. 3). A propos de cette dernière observation, il convient d'en souligner la signification biologique : elle démontre en effet que la reproduction du parasite par auto-fécondation intervient, au moins dans le cas d'infestations isolées, comme mode habituel de reproduction dans le cycle du Digène. Précisons au surplus que les œufs pondus par les parasites isolés donnent naissance à des miracidiums infestants.

Pour des populations de 5 et de 75 individus, la productivité est respectivement de 950 o.p.j. et de 830 o.p.j.

Au plan qualitatif, les courbes de productivité relatives aux différentes densités de population parasitaire offrent des caractéristiques identiques à celles signalées dans notre expérience témoin :

- le démarrage de la ponte a lieu au $9^{\mathrm{e}}$ jour d'infestation ;

- la productivité des parasites peut varier énormément d'un jour à l'autre ; 


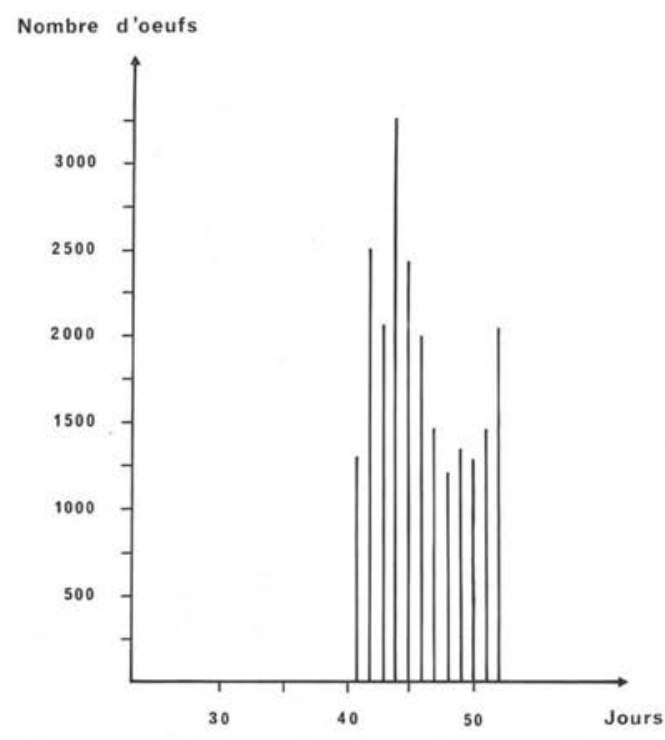

FIG. 3. - E. togoensis. Production journalière par parasite (expérience réalisée à partir d'une Souris infestée par un seul parasite).

— la courbe de productivité rend toujours compte de périodes de forte productivité alternées avec des périodes de faible productivité.

Pour les doses infestantes testées ci-dessus (jusqu'à 150 métacercaires), nous n'avons pas noté de mortalité anormale des Souris, ni constaté d'effets évidents sur la croissance et la reproduction des hôtes.

Par contre, des doses supérieures ou égales à 200 métacercaires se révèlent très fortement pathogènes pour la Souris. A partir du $30^{\mathrm{e}}$ jour, les animaux entrent en état de cachéxie jusqu'à la mort qui survient en moyenne pour tous les individus entre le $30^{\mathrm{e}}$ et le $45^{\mathrm{e}}$ jour.

Les données fournies pour nos recherches sur la productivité de $E$. togoensis chez le Vertébré d'une part, et sur sa pathogénicité d'autre part, sont de nature à permettre une optimisation de cette productivité. L'optimisation consiste à rechercher la production maximale d'œufs compatible avec la santé et la survie de la Souris. L'analyse des résultats exposés plus haut nous conduit à considérer que la population parasitaire optimale se situe aux environs de 75 parasites pour une production qui sera de l'ordre de 62000 œufs par Souris et par jour.

\section{Discussion}

L'utilisation des Trématodes dans le contrôle biologique des Bilharzioses repose sur la propriété de beaucoup d'entre eux de se développer dans la glande génitale des Mollusques vecteurs. Le développement du parasite peut conduire à une stérilisation totale du Mollusque associée à des désordres métaboliques souvent irréversibles par 
perte de la fonction digestive. A cet égard, les résultats expérimentaux obtenus avec E. togoensis sont particulièrement spectaculaires. Les effets pathogènes qui peuvent être exploités dans le cadre de la lutte biologique contre $B$. pfeifferi sont :

- une stérilisation définitive des Mollusques infestés ;

— une mortalité du vecteur lorsqu'il y a infestation avec plus de 5 miracidiums ;

- une compétition des rédies de E. togoensis avec les sporocystes de S. mansoni qui sont très rapidement détruits.

De plus, E. togoensis associe à ce pouvoir pathogène une très grande facilité de maintenance de son cycle en laboratoire et une très grande productivité qui autorisent une production en masse d'œufs :

- le parasite évolue seulement chez deux hôtes (la Souris et le Planorbe) ce qui rend la maintenance techniquement facile et peu coûteuse ;

- la longévité importante du parasite chez la Souris (6 mois) diminue la fréquence des manipulations nécessaires à l'entretien du cycle ;

- la productivité très élevée du parasite adulte peut assurer, avec un nombre relativement modeste de Souris, une production d'œufs journalière très importante ( 10 Souris infestées avec 75 métacercaires chacune assurent une production journalière de 620000 œufs en moyenne).

Par l'ensemble des caractères que nous venons d'énumérer, $E$. togoensis répond à la définition donnée par Combes (1981) du Trématode idéal pour une utilisation en lutte biologique : " espèce fortement stérilisante, fortement dominante, à forte productivité en œufs, s'enkystant chez des Mollusques et devenant adulte chez les Rongeurs de laboratoire n. Son utilisation en lutte biologique ne peut néanmoins être envisagée qu'après une étude complète des capacités de localisation des Planorbes par les miracidiums. Un programme de recherche sur ce problème est actuellement en cours au laboratoire.

Remerciements. Cette recherche a reçu le support financier du Programme spécial PNUD - Banque Mondiale - OMS de Recherche et de Formation concernant les Maladies Tropicales et du C.N.R.S. (ERA 915).

\section{BIBLIOGRAPHIE}

Cheng T. C., YeE H. W. : Histochemical demonstration of aminopeptidase activity associated with the intramolluscan stages of Philophthalmus gralli Mathis et Léger. Parasitology, 1968, s8, 473-480.

Combes C. : Trematodes : antagonism between species and sterilizing effects on snails in biological control. Parasitology, 1982, 84, 30-51.

Jourdane J., Kulo S. D. : Étude expérimentale du cycle biologique de Echinostoma togoensis n. sp., parasite à l'état larvaire de Biomphalaria pfeifferi au Togo. Ann. parasitol. Hum. Comp., 1981, 56, 477-488.

Sturters J. F. : Development of Trichobilharzia ocellata in Lymnaea stagnalis and the effects of infection on the reproductive system of the host. $Z$. Parasitenkde, I981, 64, 303-319. 